\title{
MUC1 and MUC2 mucins in flat and polypoid colorectal adenomas
}

\author{
Y Ajioka, H Watanabe, J R Jass
}

\begin{abstract}
Aims-To examine the expression of MUC1 and MUC2 apomucins and distribution of MUC phenotypes (MUC2+l MUC1-, MUC2+/MUC1+, MUC2-I MUC1+, MUC2-IMUC1-) in colorectal tubular adenomas in order to compare the distribution of phenotypes in flat and polypoid adenomas.

Methods-Endoscopically resected specimens of 35 flat and 15 polypoid tubular adenomas measuring less than $10 \mathrm{~mm}$ were examined and compared for the expression of MUC1 (MUSE11) and MUC2 (CCP58) and combined MUC phenotype distribution using conventional immunohistochemistry.

Results-There was no significant difference between flat and polypoid adenomas in their expression of MUC1 and MUC2 and the MUC phenotype distribution when stratified by grade of histological atypia. Adenomas with low grade atypia showed more extensive MUC2 expression than MUC1 (MUC2+/MUC1- phenotype). Expression of MUC1 was more extensive in adenomas with high grade atypia and the majority displayed either $\mathrm{MUC2}+1$ MUC1+ or MUC2-IMUC1+ phenotypes. Conclusions-MUC2/MUC1 phenotypes were similar in flat and polypoid adenomas when stratified by grade of atypia. High grade atypia was characterised by reduced MUC2 and increased MUC1 expression in both types of adenoma. The phenotype MUC2-IMUC1+ occurs in tubular adenomas and cannot be a specific marker for de novo colorectal cancer. (F Clin Pathol 1997;50:417-421)
\end{abstract}

Department of Pathology, Medical School, University of Queensland,

Queensland, Australia J R Jass

First Department of Pathology, School of Medicine, Niigata University, Niigata, Japan

Y Ajioka

$\mathrm{H}$ Watanabe

Correspondence to: Professor J R Jass,

Department of Pathology, Medical School, University of Queensland, Herston,

Queensland 4006, Australia.

Accepted for publication 25 February 1997 frequently seen in polypoid adenomas, is uncommon in flat adenomas. ${ }^{78}$ The adenomacarcinoma sequence encompasses a "classical or polypoid adenoma-carcinoma sequence" 19 and a "flat adenoma-carcinoma sequence". ${ }^{410}$ It has been suggested that de novo cancers could arise through the flat adenomacarcinoma sequence, ${ }^{10-12}$ as indicated by the rare occurrence of K-ras mutations in de novo cancers $^{1314}$ as well as in flat adenomas.

Genes coding for the protein backbone or apomucin component of epithelial mucins have been cloned and the expression of MUC1, MUC2, MUC3, MUC4, MUC5AC, and MUC5B has been studied in relation to the adenoma-carcinoma sequence of the colorectum. ${ }^{15-17}$ These studies have supported the concept of evolution of carcinoma through adenoma, but have hinted at alternative pathways also. Upregulation of MUC2 and neo-expression of MUC5AC accompanies the villous adenoma-mucinous carcinoma sequence, ${ }^{15}{ }^{17}$ whereas de novo cancer appears to be characterised by loss of MUC2. ${ }^{15} \mathrm{We}$ suggested previously that the phenotype MUC2-/MUC1+ might characterise the de novo pathway. ${ }^{18}$

MUC2 is regarded as the principal secretory mucin in the colorectum and is specific to goblet cells. ${ }^{19}$ Goblet cell differentiation can therefore be followed through various evolutionary pathways using MUC2 markers. MUC1 is a non-secretory glycoprotein expressed along the apical membrane of columnar cells and upregulated in various adenocarcinomas. ${ }^{18}$ The MUSE11 epitope is demonstrated in normal colorectum only following unmasking (deglycation) using periodate oxidation. Even then, MUSE11 (MUC1) staining is limited to the apical membrane of crypt base cells. ${ }^{18}$ MUC1 expression by normal colorectum has been confirmed by northern blotting ${ }^{20}$ and in situ hybridisation. ${ }^{17}$ In colorectal carcinoma, the MUSE11 epitope (MUC1) does not appear to be distributed with secretory mucin but in association with columnar cells (glycocalyx and diffusely in cytoplasm) and intracytoplasmic lumina. ${ }^{21}$ For these reasons we have proposed that MUC1 (specifically the epitope demonstrated by MUSE11) serves as a marker of cells of columnar lineage. ${ }^{21}$ Others describe a weak cytoplasmic MUC1 signal in all epithelial cells of normal colorectum using in situ hybridisation but do not provide details of localisation. ${ }^{17}$ In this study, the premise that the phenotype MUC2-/MUC1+ might characterise de novo colorectal cancer is explored in a series of polypoid and flat adenomas and using epitopes recognised by MUSE11 (MUC1) ${ }^{22}$ and CCP58 (MUC2). ${ }^{23}$

\section{Methods}

Formalin fixed, paraffin embedded tissue of 35 flat type and 15 polypoid type sporadic tubular adenomas resected endoscopically were studied. All measured less than $10 \mathrm{~mm}$. The flat 
Table 1 Number, size, and grade of atypia of flat and polypoid adenomas

\begin{tabular}{lllll}
\hline & & \multicolumn{2}{l}{ Srade of atypia } & \\
\cline { 4 - 5 } & Number & mean $(S D)$ & $L$ & $L+H$ \\
\hline Flat type & 35 & $6.0(1.8)$ & 25 & 10 \\
Polypoid type & 15 & $6.7(1.8)$ & 10 & 5 \\
\hline
\end{tabular}

L, composed of pure low grade atypia; $\mathrm{L}+\mathrm{H}$, composed of low and high grade atypia.

type was defined as follows: (1) the thickness of the mucosa was not more than twice that of the surrounding normal mucosa (exclusive of transitional mucosa immediately adjacent to adenoma), and (2) there was a flat or slightly concave surface. They included macroscopically slightly depressed or elevated lesions. The

A
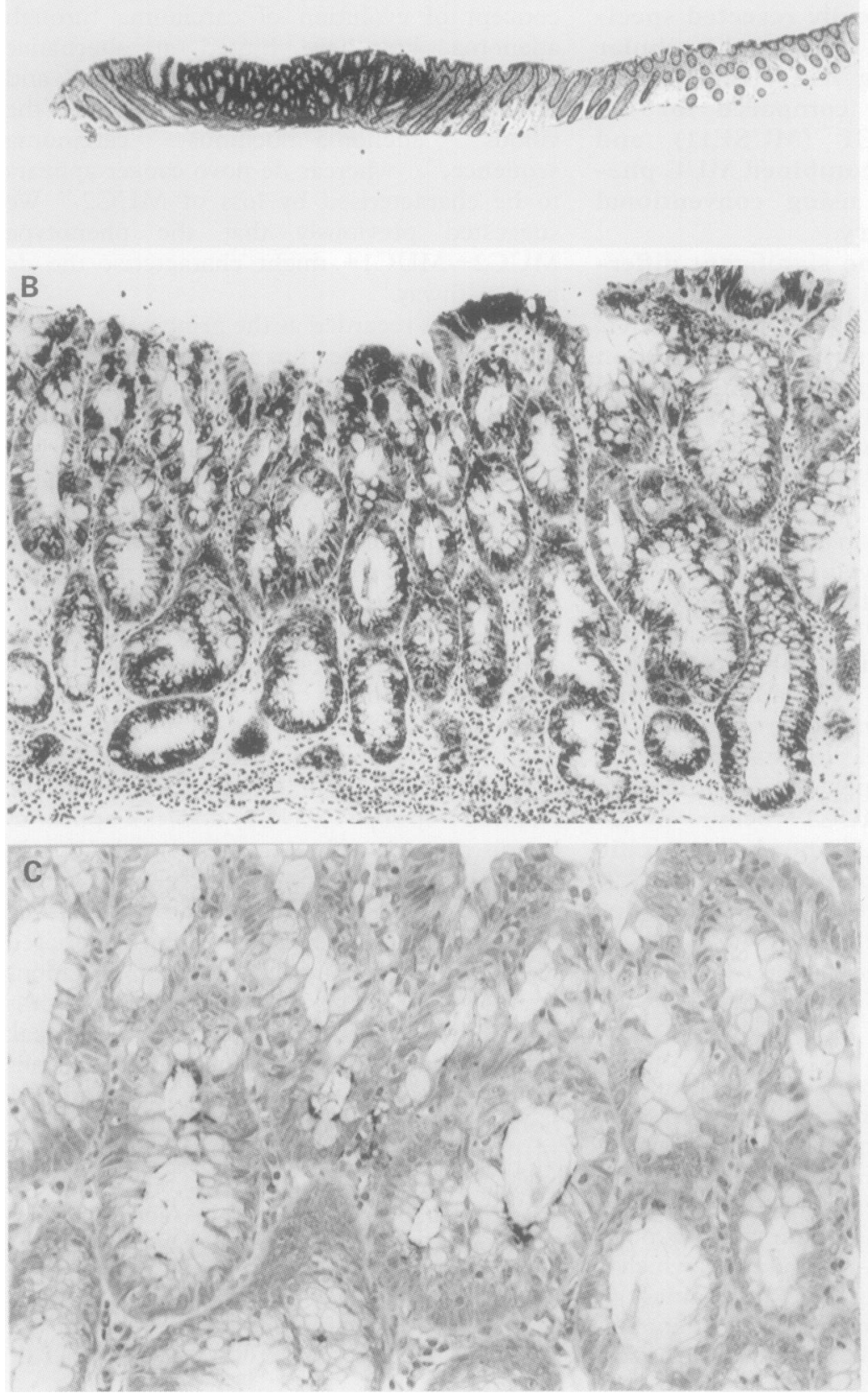

Figure 1 (A) Whole mount view of flat type adenoma (almost totally flat) with low grade atypia, measuring $3 \mathrm{~mm}(\times 5)$. (B) Diffuse and strong expression of MUC2 in perinuclear cytoplasm of cells of goblet lineage. Immunoperoxidase with CCP58 (×40). (C) Only a minute trace MUC1 expression at the apical membrane of adenomatous cells. Immunoperoxidase with MUSE11 ( 180$)$. polypoid type was either pedunculated or subpedunculated. Histological grade of atypia of adenomas was classified into low and high grade, ${ }^{24}$ equivalent to mild and moderate dysplasia, respectively, of the WHO classification. ${ }^{25}$ Table 1 shows the average size and grade of atypia of the samples. All adenomas were composed mainly of low grade atypia. Foci of high grade atypia were present in 10 flat and five polypoid-type adenomas.

Sections of $3 \mu \mathrm{m}$ thickness were cut and mounted on poly-L-lysine coated slides. These were stained with haematoxylin and eosin and with monoclonal antibodies to MUC1 (MUSE1 1, undiluted supernatant) ${ }^{22}$ and MUC2 (CCP58, ascites, diluted 1 in 1000). ${ }^{23}$ Immunostaining was visualised by the stepwise addition of biotinylated antimouse antibody followed by streptavidin-biotin peroxidase labelled complex (Dako, Glostrup, Denmark) and finally developed with diaminobenzidine. Immunostaining for MUC1 was preceded by routine periodate oxidation. ${ }^{26}$ Known positive and negative tissue controls were included with each staining run.

The extent of MUC1 and MUC2 reaction was scored as: 0,1 ( $<5 \%$ of cells), 2 ( $5-30 \%$ of cells), 3 (30-60\% of cells), or 4 (> $60 \%$ of cells). Using these scores, four MUC phenotypes were defined in which a score of at least 3 was regarded as positive: MUC2+/MUC1-, MUC2+/MUC1+, MUC2-/MUC1+, and MUC2-/MUC1-. ${ }^{18}$ MUC1 and MUC2 scores and MUC phenotypes were correlated with histology in an adjacent tissue section. For the statistical analysis, the Mann-Whitney U test was performed to compare the average MUC1 and MUC2 scores, and the $\chi^{2}$ test or Fisher's exact test were used for comparison of the MUC phenotype distribution.

\section{Results}

MUC1 was expressed along the apical membrane of adenomatous cells. In adenomas with low grade atypia (figs 1 and 2), the reactivity was limited to a minute trace and confined to the upper part (fig 1C) or the basal part of the crypt (fig $2 \mathrm{C}$ ). In adenomas with high grade atypia (fig 3), MUC1 was expressed diffusely along the entire crypt (fig 3B). MUC2 was demonstrated in the perinuclear cytoplasm of cells with a goblet cell type theca and with apical mucous droplets (figs 1B, 2B, 3A). MUC2 distribution within crypts showed no relation to histological grade of atypia. No MUC2 expression was observed within extracellular material.

The average MUC2 and MUC1 scores in adenomas with low grade atypia were 3.4 and 1.9 for flat type and 3.6 and 2.3 for polypoid type (fig 4). MUC2 scores were higher than MUC1 regardless of the macroscopic type. Of 50 adenomas with low grade atypia, 46 (92\%) showed MUC2 scores of 3 or more, while for MUC1 these scores were detected in 21 $(42 \%)$. MUC2+/MUC1 - was the major phenotype, comprising $60 \%(21 / 35)$ and $53 \%$ $(8 / 15)$ of flat type and polypoid type adenomas (fig 5). In adenomas with high grade atypia, the 

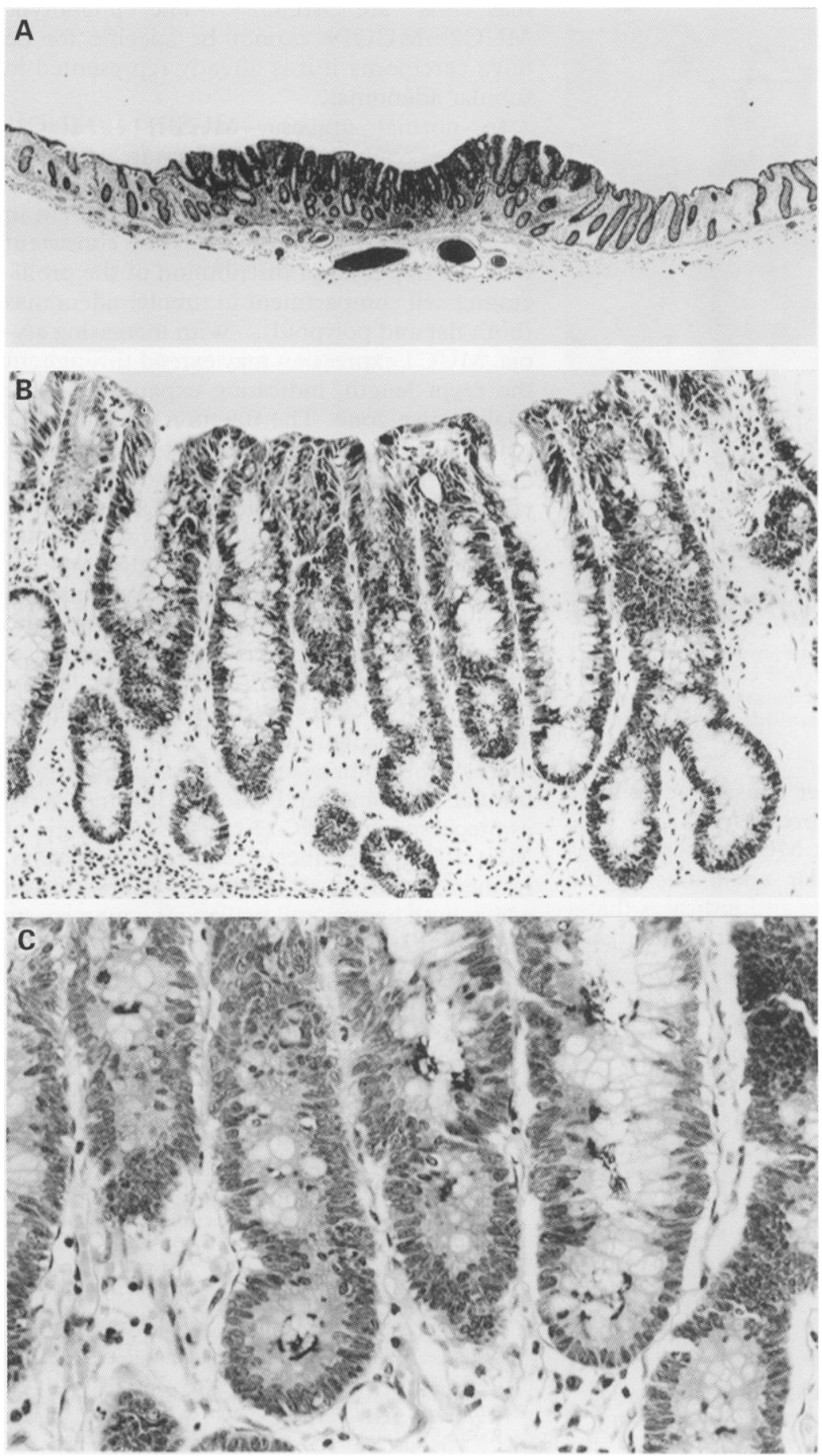

Figure 2 (A) Whole mount view of flat type adenoma (elevated lesion with slight concave surface) with low grade atypia, measuring $5 \mathrm{~mm}(\times 5)$. (B) Diffuse expression of MUC2. Immunoperoxidase with CCP58 ( $\times 50)$. (C) MUC1 expression is seen at the base of the crypt. Immunoperoxidase with MUSE11 (×100).

average MUC2 and MUC1 scores in the areas of high grade atypia were 2.4 and 3.4 for flat and 2.0 and 3.3 for polypoid types (fig 4) (the MUC1 score exceeding that of MUC2). Of 15 adenomas with high grade atypia, $13(86.7 \%)$ and nine $(60 \%)$ were scored (in foci of high grade atypia) as 3 or more for MUCl and MUC2, respectively. MUC2+/MUC1+ and MUC2-/MUC1+ were the predominant phenotypes in foci of high grade atypia (fig 5 ). The phenotype MUC2-/MUC1- was not observed in any specimen.

There was no significant difference in either average MUC scores or the distribution of MUC phenotypes in flat and polypoid type adenomas when stratified by areas of low grade and high grade atypia.

\section{Discussion}

The epitopes recognised by MUSE11 (MUC1) and CCP58 (MUC2) in colorectal epithelium are assumed to be specific for columnar and goblet cell lineages, respectively. ${ }^{18}{ }^{21}$ These cell lineages were demonstrated immunohistochemically in colorectal cancers, which were then classified into four phenotypes according to the extent of MUC2 and MUC1 expression: MUC2+/MUC1-, MUC2+/MUC1+, MUC2-/MUC1+, and MUC2-/MUC1-. ${ }^{18}$ Absence of expression of a particular apomucin could mean that the associated cell type is not represented (for example absence of goblet cells in MUC2-/ MUC1 + cancers) or simply that expression has not occurred in a cancer that nevertheless includes the relevant cell type (for example no appreciable MUC1 in association with columnar cells).

MUC1, MUC2, MUC3, MUC4, and MUC5 expression has been investigated in colorectal adenomas previously. ${ }^{16} 17$ MUC1, MUC2, and MUC3 show increasing immunoreactivity in association with polyp size, villosity, and degree of dysplasia. ${ }^{16}$ There is neoexpression of MUC5AC in villous adenomas whereas MUC5B is not expressed at all. ${ }^{17}$ The present study showed increased MUC1 and reduced MUC2 expression in foci of high grade atypia in both flat and polypoid tubular adenomas. The progressive increase in MUC1 expression may not represent upregulation since MUC1 mRNA is not necessarily increased in colorectal neoplasia. ${ }^{1620}$ Increased immunostaining in neoplasms may reflect reduced glycosylation (unmasking) of the apomucin or simply an accumulation that reflects the increased longevity of a transformed and relatively immortalised epithelium. The reduction of MUC2 in foci of high grade atypia is consistent with the well documented loss of mucin production occurring in parallel with increasing dysplasia, but was not shown in an earlier study. ${ }^{16}$ Increased MUC2 immunoreactivity in association with degree of dysplasia ${ }^{16}$ may be explained by the confounding effect of villosity. Villous adenomas often secrete abundant mucin but are also prone to develop high grade dysplasia. MUC2 expression is increased in villous adenomas and mucinous carcinomas, ${ }^{15} 16$ an observation that fits with the histogenetic sequence linking villous adenoma to mucinous adenocarcinoma. The different findings of the present study are explained by the exclusion of villous adenomas.

Despite their contrasting molecular backgrounds, this study showed flat and polypoid adenomas to be similar in their phenotypic profiles with respect to MUC1 and MUC2. Tubular adenomas, whether polypoid or flat, may with increasing atypia display the phenotype MUC2-/MUC1+. This phenotype has been linked to de novo carcinoma of the large intestine. ${ }^{1518}$ It has been suggested that expression of MUC2 indicates cancer histogenesis through the adenoma-carcinoma 


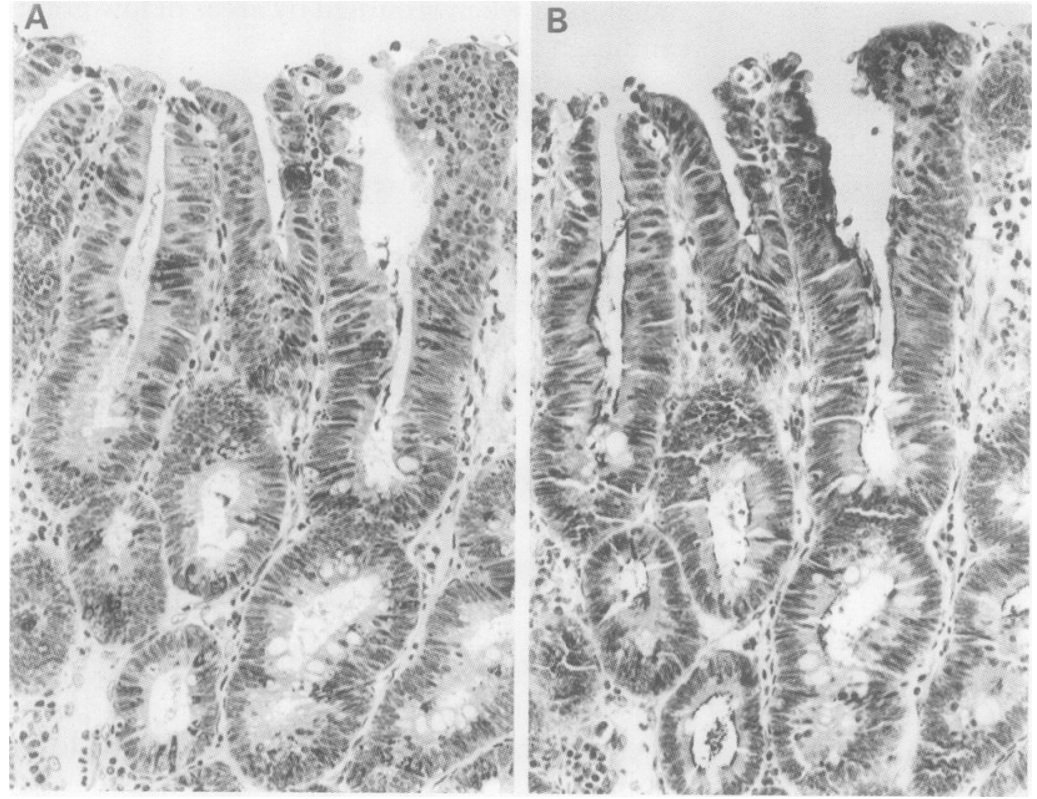

Figure 3 (A) MUC2 expression within adenoma with high grade atypia. Reactive cells are diffusely distributed but small in number. Immunoperoxidase with CCP58 (×62).

(B) MUC1 expression of the identical field to $(A)$. Reactivity at the apical membrane is strong and diffuse along the entire adenomatous crypt. Immunoperoxidase with MUSE11 (×62).

sequence, whereas cancer considered to arise de novo does not express MUC2. ${ }^{15}$ The present study shows that MUC2 expression is downregulated in tubular adenomas at the stage of high grade atypia and indicates that a proportion of carcinomas may arise in adeno-

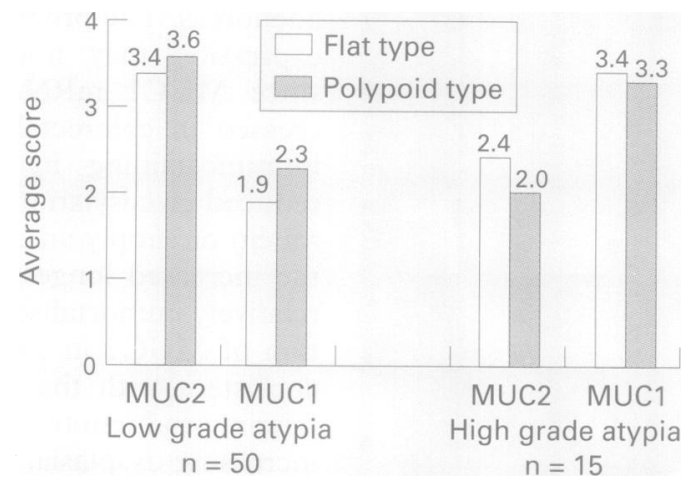

Figure 4 Average MUC1 and MUC2 staining scores for flat and polypoid type adenomas by histological grade of atypia.
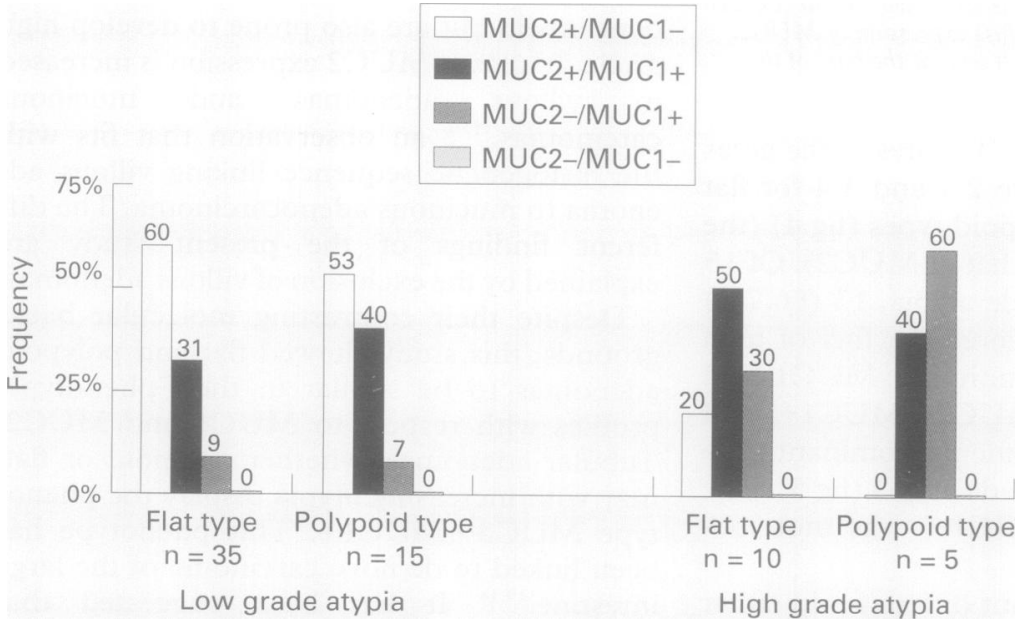

Figure 5 Frequency of four MUC phenotypes for flat and polypoid type adenomas by histological grade of atypia. mas that are MUC2-. The phenotype MUC2-/MUC1+ cannot be specific for de novo carcinoma if it is already represented in tubular adenomas.

In normal mucosa, MUSE11 (MUC1) staining is restricted to the immature columnar cells of the lower crypt. ${ }^{18}$ In adenomas, staining is increased and in a subset is more evident in superficial locations. This would be consistent with the upper crypt distribution of the proliferating cell compartment in tubular adenomas (both flat and polypoid). ${ }^{27}$ With increasing atypia, MUC1 expression may extend throughout the crypt length, indicating expansion of the proliferative zone. The function of MUC1 or episialin remains obscure, but it seems to inhibit cadherin mediated adhesion between epithelial cells in cell culture. ${ }^{28}$ Therefore loss of apical polarity could lead to cellular discohesion while inappropriate glycosylation may allow malignant cells to attach themselves to endothelium, a critical step in the metastatic cascade. Specifically, aberrantly expressed SLe ${ }^{x}$ serves as a ligand for E-selectin ${ }^{29}$ and breast mucin MUC1 serves as a ligand for ICAM-1. ${ }^{30}$ This would explain why MUC1+ cancers are more prone to metastasis. ${ }^{18}$ If flat and polypoid tubular adenomas and villous adenomas serve as precursors of MUC1+ cancers, the origin of MUC1 negative cancers requires explanation. Is there a subset of colorectal neoplasms in which both benign and malignant counterparts share a normal (MUC2+/MUC1-) phenotype? We are now exploring the possibility that this phenotype would apply to serrated neoplasms.

We thank Dr Y Hinoda and Dr I F C McKenzie for their gifts of MUSE11 and CCP58 monoclonal antibodies.

1 Morson BC, Dawson IMP. Adenomas and the adenomacarcinoma sequence. In: Morson BC, Dawson IMP, eds. Gastrointestinal pathology, 2nd ed. Oxford: Blackwell, 1979.

2 Rickert R, Auerbach O, Garfinkel L. Adenomatous lesions of the large intestine. Cancer 1973;31:1260-70.

3 Hamilton SR. Pathogenesis of polyps (adenomas). Dis Colon Rectum 1983;26:413-14.

4 Muto T, Kamiya J, Sawada T, Konishi F, Sugihara K, Kubota Y, et al. Small "flat adenoma" of the large bowel with special reference to its clinicopathological features. Dis Colon Rectum 1985;28:847-51.

5 Wolber RA, Owen DA. Flat adenomas of the colon. Human Pathol 1991;22:70-4.

6 Karita M, Cantero D, Okita K. Endoscopic diagnosis and resection treatment for flat adenoma with severe dysplasia. Am $\mathcal{f}$ Gastroenterol 1993;88:1421-3.

7 Fujimori T, Satonaka K, Yamamura-Idei Y, Nagasako K, Maeda S. Non-involvement of ras mutation in flat colorec tal adenomas and carcinomas. Int 7 Cancer 1994;57:51-5.

8 Yamagata S, Muto T, Uchida Y, Masaki T, Sawada T, Tsuno $\mathrm{N}$, et al. Lower incidence of K-ras codon-12 mutation in flat colorectal adenomas than in polypoid adenomas. $\mathcal{f} p n \mathcal{F}$ Cancer Res 1994;85:147-51.

9 Muto T, Bussey HJ, Morson BC. The evolution of cancer of the colon and rectum. Cancer 1975;36:2251-70.

10 Adachi $M$, Muto T, Morioka Y, Ikenaga T, Hara M. Flat adenoma and flat mucosal carcinoma (IIb type) - a new precursor of colorectal carcinoma? Dis Colon Rectum 1988; 31:236-43.

11 Minamoto $T$, Sawaguchi $K$, Ohta $T$, Itoh $T$, Mai $M$ Superficial-type adenomas and adenocarcinomas of the Superficial-type adenomas and adenocarcinomas of the
colon and rectum: a comparative morphological study.

12 Owen DA. Flat adenoma, flat carcinoma, and de novo carcinoma of the colon. Cancer 1996;77:3-6.

13 Aoki T, Takeda S, Yanagisawa A, Kato Y, Ajioka Y, Watanabe $\mathrm{H}$, et al. APC and 53 mutations in de novo colorecta adenocarcinomas. Hum Mutat 1994;3:342-6.

14 Yukawa M, Fujimori T, Maeda S, Tabuchi M, Nagasako K. Comparative clinicopathological and immunohistochemical study of ras and 553 in flat and polypoid type colorecta tumours. Gut 1994;35:1258-61.

15 Blank M, Klussmann E, Kruger-Krasagakes S, SchmittGraff A, Stolte M, Bornhoeft $G$, et al. Expression of 
MUC2-mucin in colorectal adenomas and carcinomas of different histological types. Int $\mathcal{F}$ Cancer 1994;59:301-6.

16 Ho SB, Ewing SL, Montgomery CK, Kim YS. Altered mucin core peptide immunoreactivity in the colon polyp-carcinoma sequence. Oncol Res 1996;8:53-61.

17 Buisine M-P, Janin A, Maunoury V, Audié J-P, Delescau M-P, Copin M-C, et al. Aberrant expression of a human mucin gene (MUC5AC) in rectosigmoid villous adenoma. Gucin gene (MUC5AC) in recto

18 Ajioka Y, Allison LJ, Jass JR. Significance of MUC1 and MUC2 mucin expression in colorectal cancer. $f$ Clin Patho 1996;49:560-4.

19 Tytgat KMAJ, Büller HA, Opdam FJM, Kim YS, Einerhand AWC, Dekker J. Biosynthesis of human colonic mucin Muc2 is the prominent secretory mucin. Gastroenterology 1994;107:1352-63.

20 Ogata S, Uehara H, Chen A, Itzkowitz SH. Mucin gene expression in colonic tissues and cell lines. Cancer Res 1992;52:5971-8.

21 Ajioka Y, Xing P-X, Hinoda Y, Jass JR. Correlative histochemical study providing evidence for the dual nature histochemical study providing evidence for the dual nature
of human colorectal cancer mucin. Histochem $₹$ 1997;29:1of hum

22 Hinoda Y, Nakagawa N, Ohe Y, Kakiuchi H, Tsujisaki M, Imai $\mathrm{K}$, et al. Recognition of the polypeptide core of mucin by monoclonal antibody MUSE11 against an adenocarcinoma-associated antigen. $f_{p n} \mathcal{f}$ Cancer Res 1990;81:1206-9.
23 Xing P-X, Prenzoska J, Layton GT, Devine PL, McKenzie IFC. Second generation monoclonal antibodies to intestinal MUC2 peptide reactive with colon cancer. 7 Natl Cancer Inst 1992;84:699-703.

24 Watanabe H, Ajioka Y, Yamaguchi M, Noda Y, Honma T, Motoyama T. Histological criteria of colorectal adenomas and carcinomas [in Japanese with English abstract]. Stomand carcinomas [in Japan

25 Jass JR, Sobin LH, eds. Histological typing of intestinal tumors, 2nd ed. Berlin: Springer-Verlag, 1989.

26 Pearse AGE. Histochemistry-theoretical and applied, 3rd ed Pearse AGE. Histochemistry theoretical and app
vol 1. London: Churchill Livingstone, 1968.

27 Kobayashi M, Watanabe H, Ajioka Y, Honma T, Asakura H Proliferating cell nuclear antigen/cyclin expression in smal adenomas of the large intestine in relation to size and macroscopic appearance. $f$ Gastroenterol 1994;29:139-46.

28 Wesseling J, van der Valk SW, Hilkens J. A mechanism for inhibition of E-cadherin-mediated cell-cell adhesion by the min episialin/MUC1. Mol Biol Cell 1996;7:565-77.

29 Nakagawa T, Fukushima K, Hirota M, Kusano H, Ayabe $H$ Tomita $\mathrm{M}$, et al. Immunohistochemical expression of Sialyl $\mathrm{Le}^{\mathrm{x}}$ antigen in relation to survival of patients with colorec$\mathrm{Le}^{\mathrm{x}}$ antigen in relation to survival of patient
tal carcinoma. Cancer 1993;72:2323-30.

30 Regimbald LH, Pilarski LM, Longenecker M, Reddish MA Zimmermann G, Hugh JC. The breast mucin MUC1 as a novel adhesion ligand for endothelial intercellular adhesion meolcule 1 in breast cancer. Cancer Res 1996;56:4244-9. 